\title{
The mediating role of emotional intelligence between spiritual intelligence and mental health problems among Iranian adolescents
}

\begin{abstract}
This study examined the mediating role of emotional intelligence between spiritual intelligence and mental health. The participants in the study were 247 high school Iranian students, (124 male and 123 female, in the age range between 15 and 17 years old). The results showed that spiritual and emotional intelligences explained mental health problems differently. Structural equation modelling revealed that spiritual intelligence had indirect effect on mental health problems, via emotional intelligence. These findings have implications for prevention of mental health problems among adolescent.
\end{abstract}

Keyword: Spiritual intelligence; Emotional intelligence; Adolescents; Mental health 